\title{
The New Design of an Acousto-Optic Device with a Mobile Power Pack
}

\author{
Yi-Yu Lu' ${ }^{1}$ Wen-Bin Lin, Zheng-Ying Li \\ Department of Electrical Engineering, Far-East University \\ No. 49, Zhonghua Rd., Xinshi Dist., Tainan City, 74448, Taiwan \\ E-mail:yiyulu@cc.feu.edu.tw
}

\begin{abstract}
The new designed acousto-optic device has adjustable, it is 1.5 times to extend its length. Fitting plastic optic fiber into transparent tube, the path of light can be increased and the light area will be enlarged. The device can present different color mode of light with RGB and high light LEDs as the light source. Adding the USB charge and discharge function into the device, make it has the function of mobile power pack.
\end{abstract}

Keywords: Multifunction, acousto-optic warning device, mobile power pack.

\section{Introduction}

Traffic batons plays an important role in policemen daily works. Their accoutrements also need a flashlight and whistle when on duty, and thus this study aimed to integrate these into one device. This work thus presents the design of a single apparatus with the functions of a glow stick, warning system and camping light in the form of an acousto-optic warning device. The multifunctional acousto-optic device with a mobile power pack will be introduced for the demand of amusement and convenient consideration.

A typical flashlight is constructed from a light bulb and focusing mirrors, and over the last twenty years light emitting diodes (LEDs) have been applied on more lighting devices, as they can reduce electricity consumption and have a long lifecycle. Flashlight manufacturers are also facing the product innovation and orientation problem and how to improve the applications and extend the product life-cycle. Moreover, since smartphones can also work as flashlights, the flashlight industry is facing a crisis of falling sales. Further investigate the destruction of mobile phone innovation, just like the original online music under carrier service arise, causing the collapse of the traditional record industry is facing the same crisis [1]. Today, the mobile phone also undermine innovation in many industries began to face a crisis of survival. Flashlight if not actively seek to change, they may face the fate of being eliminated.

This study thus presents the design of a multifunctional acousto-optic warning device with a mobile power pack using plastic optical fiber [2]. Since the late 1990s, high performance plastic optical fiber based on perfluorinated polymers began to appear on the marketplace [3-4], but it has not yet been used in flashlights. Plastic optical fiber has the advantage of bending easily and not breaking, and its falling price means that it is now used for medical and recreational purposes. The advantages of the area of the light guide path increasing are implemented for this acousto-optic device design.

Conventional flashlights usually project light in only one direction only, and while this enables of directivity 
and concentration, the relation between the light source and direction of sight is unclear, and thus people may be unsure of the location of the light source [5]. This is an issue that could be improved.

\section{Materials and methods}

The device includes five major parts: (1) adjustable focus, (2) plastic optical fiber, (3) LED driver, (4) battery, and (5) micro USB power pack. The structure of multifunction acousto-optic warning device is depicted in Fig. 1.

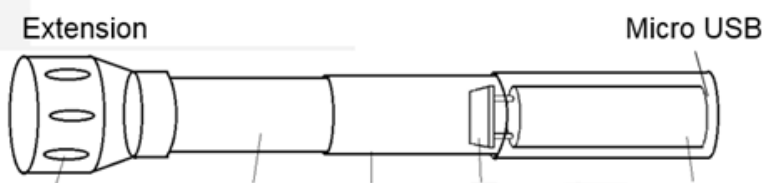

Adjustable Plastic optical fiber LED driver Li battery focus

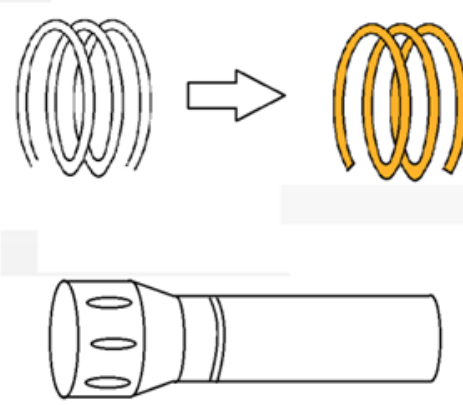

Figure 1. The multifunctional acousto-optic warning device.

\subsection{Hardware}

The basic circuit of the microcontroller unit (MCU) is shown in Fig. 2. The related program is written in Keil C.

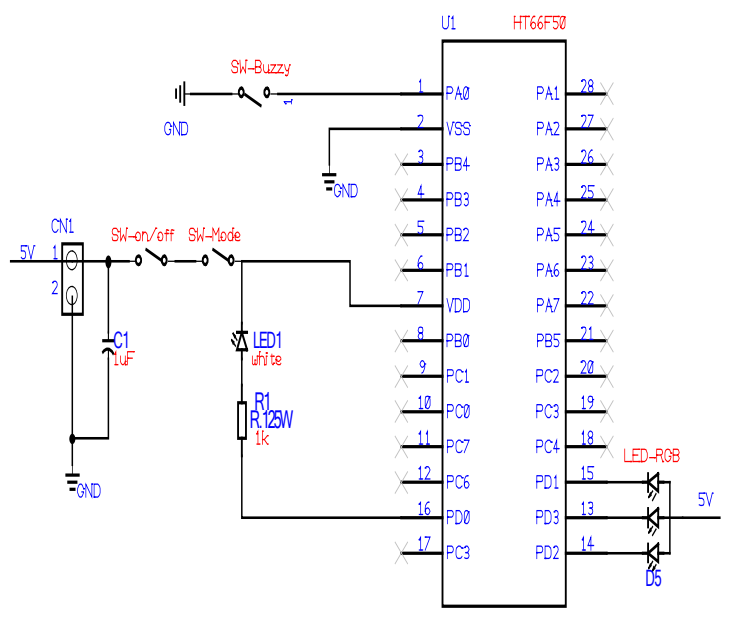

Figure 2. MCU circuit
A 555 Oscillator is a type of relaxation oscillator for generating stabilized square wave output waveforms of either a fixed frequency of up to $500 \mathrm{kHz}$ or of varying duty cycles from 50 to $100 \%$. The OP amplifier 741 is used to increase the volume. The warning function circuit is shown in Fig. 3.

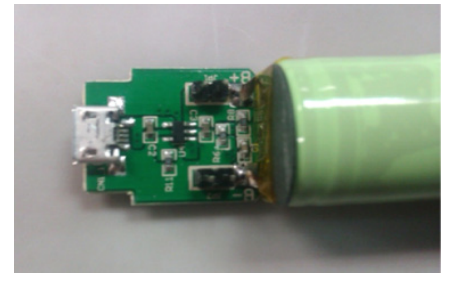

Figure 3. The warning function circuit

\subsection{USB power pack}

The USB boost and buck module are shown in Figs. 4 and 5 .

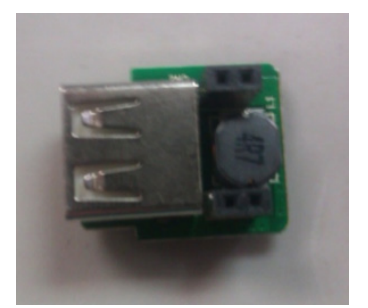

Figure 4. USB boost module

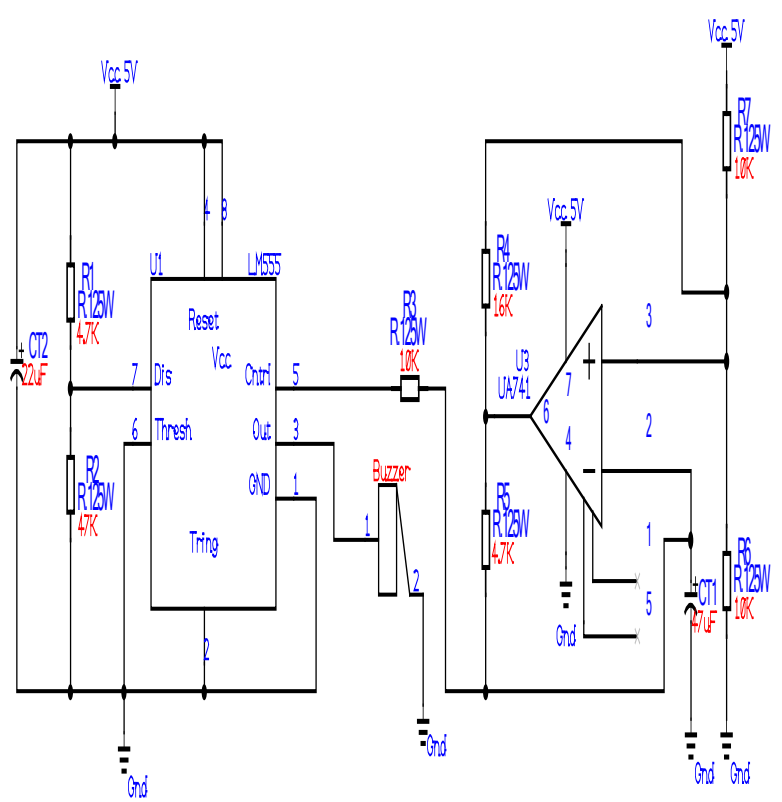

Figure 5. USB buck module 


\subsection{Software}

The flowchart and interrupt subroutine for the acoustooptical warning device are depicted in Fig. 6.

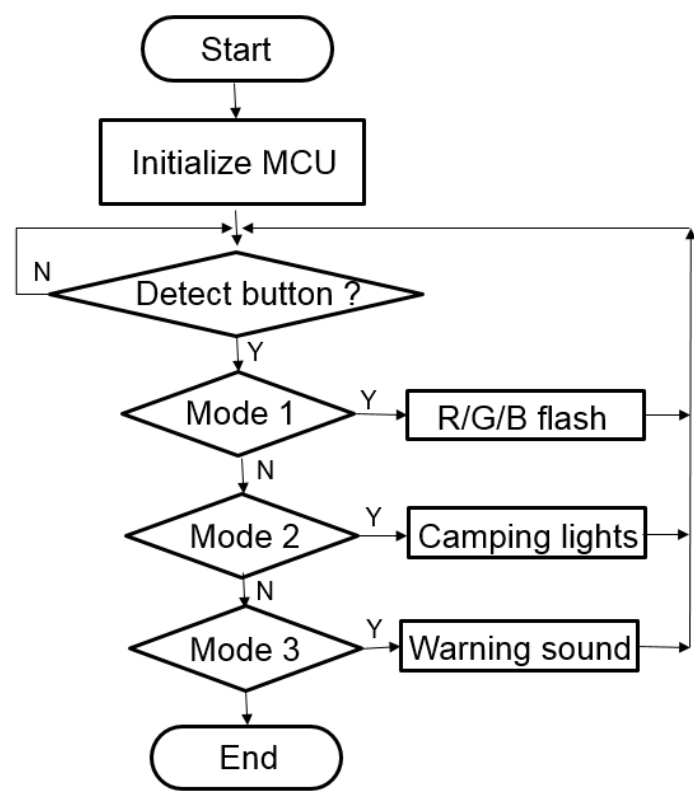

Figure 6. Flowchart for acousto-optic warning device

\section{Results and Discussion}

The device comprises three subsystems which are RGB LED, warning system and mobile power pack. Be able to give consideration to people's recreation and demand of amusement at the same time, an acousto-optic warning device with multifunction was designed. The transparent tube used in the device contains a plastic optical fiber, which is not currently seen in any similar product. The tube can increase the light guide and improve the luminous area. The device uses both RGB LEDs and high-brightness white LEDs as dual light sources, thus enabling different patterns of light and color, so the device is both functional and usable. The flashlight can be extended to 1.5 times its length, increasing the light guide and the illuminated area. In addition, the use of a USB charge and discharge function from lithium battery means that this device can also work as a mobile power pack.

The body of the flashlight is flexible, and it is convenient for use at night and for directing traffic. It combines a flashlight and a baton, and can be used when camping and to make warning sounds. On the other hands, it introduced the design of polymer optical fiber and lithium battery for energy-conservation and increasing efficiency consideration. Further, since it includes RGB LEDs, it can be used as a glow stick in concerts. The LEDs can make the light cover the whole flashlight via the optical fiber. The flashlight has an additional micro USB charging socket. The multifunctional design is the goal of various products now. This design has already been patented as a multi audible warning device at the Intellectual Property Office of Taiwan on September 21, 2014. Images of different colors of flash lighting are shown in Fig. 7.

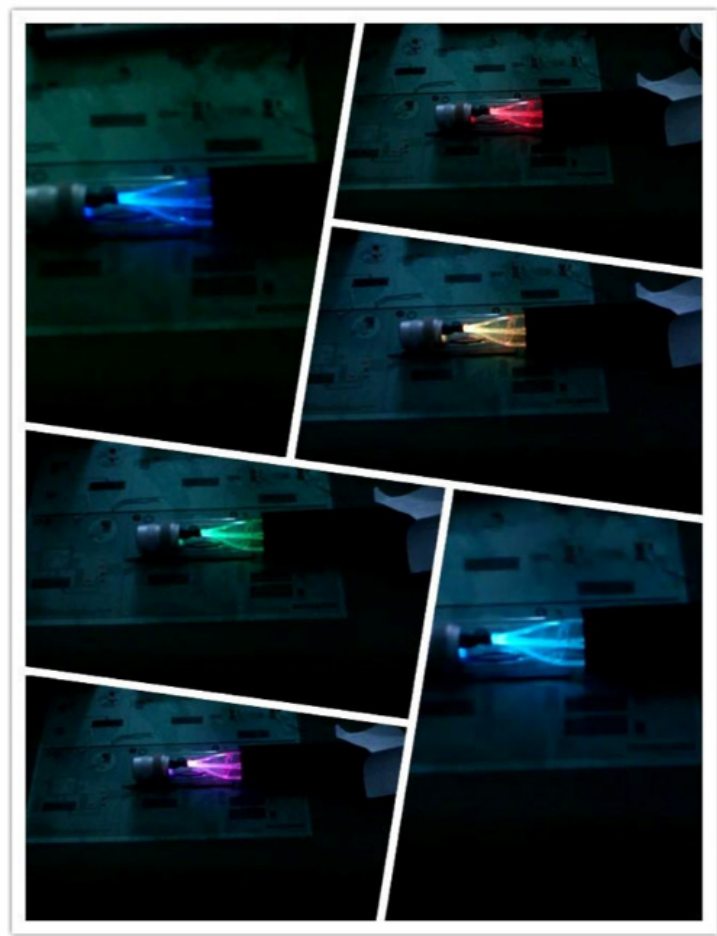

Figure 7. Photos of different colors of flash lighting

\section{Conclusions}

The design have functionality and practicability concurrently. The concept of a multifunctional acoustooptic warning device is introduced in this work. A multifunction acousto-optic warning device has obtained our country's new patent for this study. There are multifunction in this device which are as the traffic 
baton, camping light, glowing sticks in the concert and using for women's security at night, etc. The new designed acousto-optic device has adjustable, it is 1.5 times to extend its length. The advantage of this device is that when the body is exposed outside the transparent tube, the LED light source can diffuse along the light guide, and thus the lamp body can be projected in one direction, and so achieve the effect of centralized lighting. The structure also has the effect of concentrated and omni-directional diffusion of light.

\section{Acknowledgements}

This work was in part supported by the Ministry of Science and Technology, Taiwan, Republic of China, under the research grants 104-2815-C-269-018-E.

\section{References}

1. Industrial technology, vol. 283, no. 5, pp. 4-7, 2015.

2. C. M. Okonkwo, E. Tangdiongga, H. Yang, D. Visani, S. Loquai, R. Kruglov, B. Charbonnier, M. Ouzzif, I. Greiss, O. Ziemann, R. Gaudino and A. M. J. Koonen, "Recent results from the eu pof-plus project: multigigabit transmission over $1 \mathrm{~mm}$ core diameter plastic optical fibers," vol. 29., no.2., pp. 186-193, 2011.

3. S. Randel and C. Bunge, "Spectrally efficient polymer optical fiber transmission," Coherent Optical Communications, Subsystems and Systems, Proc. of SPIE, vol. 7960, 2011.

4. F. Beguin and E. Frackowiak ed., "Supercapacitors: Materials, Systems and Applications," New Jersey: Wiley, pp. 515, 2013.

5. J. Zubia and J. Arrue, "Plastic optical fibers: an introduction to their technological processes and applications," Optical Fiber Technology, vol. 7, pp. 1011402001. 